\title{
Review of Ivan Moscati's Measuring Utility: From the Marginal Revolution to Behavioral Economics. New York, NY: Oxford University Press, 2019, 326 pp.
}

\author{
ANNALISA COSTELLA \\ Erasmus School of Philosophy \\ ENRICO MATTIA SALONIA \\ Toulouse School of Economics
}

In the eighteenth century, Adam Smith and David Ricardo maintained that the exchange value of a good depends on the quantity of labor necessary to produce it. Later, during the so-called 'Marginal Revolution' in economics, it was argued that the exchange value of a good depends on the marginal utility enjoyed by an individual in the economy. This latter idea subsequently became the cornerstone of most microeconomic and, more recently, macroeconomic analyses. However, as Ivan Moscati reminds us in Measuring Utility: From the Marginal Revolution to Behavioral Economics, the main problem at the heart of the history of marginal utility, and utility more broadly, is that it cannot be observed and measured in a straightforward way (2). This has been the source of heated debates on the correct method to measure utility.

In Measuring Utility, Ivan Moscati sets out to reconstruct the history of these discussions from the 1870s, the years of the 'Marginal Revolution', to 1985, the year that allegedly marks the birth of behavioral economics. Moscati's ambitious project dovetails his prolific work in a series of prior papers ${ }^{1}$ with his most recent research on the topic. The result is a historical analysis of utility measurement placed within a reconstruction of the debate on general scientific measurement that took place at the same time (1870-1985).

The book's goal is fourfold. Firstly, it illuminates the extent to which discussions on general scientific measurement shaped how economists have conceived the measurement of utility over time. Secondly, it offers an alternative account to the dominant historical reconstruction of the

\footnotetext{
${ }^{1}$ See Moscati (2007, 2013a, 2013b, 2016a, 2016b, 2016c, 2017) for a selection of his most prominent work on utility over the years. The interested reader may further consult the complete references to Moscati's work listed in Measuring Utility.
}

AuTHORs' NotE: We would like to thank Conrad Heilmann and three anonymous referees for their constructive and extensive feedback. 
dichotomy between cardinal and ordinal utility. Thirdly, it analyses the interplay between the history of scientific measurement in general and that in economics, psychology and other disciplines in order to provide a better framework for understanding the development of utility measurement. Lastly, it presents a thorough discussion of the epistemological problems inherent to utility measurement.

\section{BACKGROUND}

Before we address the book, it is useful to recall some basic concepts from the foundations of contemporary microeconomic theory. These concepts are key to grasping Moscati's arguments and how they fit together throughout the text. In his analysis, Moscati underlines how debates about general scientific measurement influenced the prevalent utility functional forms that were adopted in every period. Every form of utility function entails an assumption about how utility itself can be measured. What follows is a summary of the main utility functional forms used in contemporary research, with explicit links between these forms and the notion of measurement that underlies them. ${ }^{2}$

A utility function, which represents how an individual ranks all options $x$ in the set of possible alternatives $X$ (the individual's preferences, in economic terms), can be either ordinal or cardinal. It is ordinal if it is unique up to any monotonic increasing transformation. This means that if $U(\cdot)$ represents the preferences of an individual over a set $X$, then $U^{\prime}(\cdot)=f(U(\cdot))$, where $f$ is a strictly increasing function, also represents the exact same preferences. Ordinal utility is measurable on an ordinal scale of measurement. The only meaningful information on such a scale is the order between the utility numbers (or other nonnumerical measures) assigned to the alternatives.

On the other hand, a utility function is cardinal if it is unique only up to linear increasing transformations. This means that $U(\cdot)$ is a cardinal utility function if and only if every linear affine transformation $U^{\prime}(\cdot)=$ $\alpha U(\cdot)+\beta$ (with $\alpha>0$ ) represents the same preferences that $U(\cdot)$ does. Cardinal utility is, instead, measurable on an interval scale of measurement. An interval scale of measurement carries meaningful information not only about the ranking of the utility numbers assigned to the respective alternatives but also about the differences between these numerical

\footnotetext{
${ }^{2}$ See, for example, the classical book by Mas-Colell, Whinston, and Green (1995).
} 
measures. ${ }^{3}$ The reader should notice that this is a richer notion of measurement than the ordinal one, as ordinal utility scales do not preserve utility differences.

Moscati introduces a third form of utility which he refers to as ratioscale utility. This latter class of utility functions constitutes a subset of cardinal utility functions. A utility function $U(\cdot)$ is ratio-scale if it is unique only up to a subset of affine linear transformations: that is, the set of proportional transformations $U^{\prime}(\cdot)=\alpha U(\cdot)$ (with $\alpha>0$ ). As the reader may notice, the parameter $\beta$ of cardinal utility functions is equal to zero. Ratioscale utility is associated with ratio-scale measurement. In addition to the ranking and the differences between numerical measures, ratio-scale utility also preserves the ratios between these measures. ${ }^{4}$ Hence, it is a notion of measurement richer than the ordinal and the cardinal one. With this technical background in mind, we can now turn to the arguments in the book.

\section{SUMMARY OF THE ARGUMENT}

Measuring Utility is organized into four parts ordered chronologically and addressing distinct periods. The first part (1870-1910) reconstructs the debates on utility theory among the first and second generation of marginalists such as William S. Jevons, Carl Menger, Léon Walras, Alfred Marshall, and Francis Y. Edgeworth. Here, Moscati argues for the novel thesis that the common notion of utility at that time was not, as it is nowadays often believed, cardinal utility. Rather, it was ratio-scale utility. To provide evidence for this, he gives a broad overview of how measurement was understood in the intellectual environment of the time. Specifically, he draws on the discussions on measurement that took place in four fields: philosophy, psychology, physics, and mathematics. In each of these fields, Moscati argues, the prevailing conception of measurement was the unit-based one. He writes that a magnitude is measurable in the unitbased sense if there is a unit of measurement that can be summed numerically. The discussions by the early marginalists were focused on what could constitute a unit of utility and on assessing utility ratios rather than utility differences. Therefore, these authors were not cardinalists in the current sense of the term. Instead, they adhered to a shared view of utility

\footnotetext{
${ }^{3}$ This means that, in addition to the condition that must be respected by ordinal measurement, cardinal measurement must respect $U(x)-U(y) \geq U(w)-U(z)$ if and only if $U^{\prime}(x)-U^{\prime}(y) \geq U^{\prime}(w)-U^{\prime}(z)$ where $U^{\prime}(v)=\alpha U(v)+\beta$ for all alternatives $v$.

${ }^{4}$ Formally, $U(x) / U(y)=\alpha U(x) / \alpha U(y)$.
} 
as ratio-scale utility. ${ }^{5}$ In fact, the utility form they had in mind did not allow for general affine transformations and, Moscati argues, this constituted a significant obstacle to the development of economic theory. The reason for this is that some of these authors, such as Walras, thought that measuring utility is necessary for performing economic analysis. However, they failed to identify what the unit of utility was and therefore believed that it was impossible to measure utility. Moscati's discussion of the solutions later developed to tackle this problem occupies the second part of the book covering the Ordinal Revolution (1910-1945).

The Ordinal Revolution is so-called because it was during that period that the Italian economist Vilfredo Pareto developed the new notion of ordinal utility, as opposed to ratio-scale utility. Pareto's claim that it is possible to rank utility ordinally was welcomed by the economic academic environment of the time. Both the first generation of Austrians, such as Ludwig von Mises and Franz Čuhel, and, later, leading economists gathered at the LSE, such as Roy G. D. Allen, Friedrich von Hayek, John Hicks, Nicholas Kaldor, and Lionel Robbins, worked on and fostered the thesis. The possibility of ranking utility indices ordinally was novel. However, how measurement was conceived in that period was still captive to past conceptions. In fact, all of the economists mentioned above, Moscati argues, still shared a unit-based understanding of measurement. Hence, there were no relevant differences between economists during the Ordinal Revolution and their predecessors-with one exception: the former abandoned the idea that the possibility of measuring utility is a necessary condition for performing economic analysis, as Walras believed.

It was only from 1945 onwards that the situation changed abruptly. This turn marks the beginning of the third part of Moscati's book, in which he analyses a brief but exciting period in economics (1945-1955). The debate that followed the 1944 publication of Theory of Games and Economic Behavior, in which John von Neumann and Oskar Morgenstern ([1944] 2007) developed a now-standard conception of expected utility, led to a profound revision of the prevalent understanding of utility measurement. The leading actors in these discussions were the economists Maurice Allais, William Baumol, Milton Friedman, Jacob Marschak, Paul Samuelson, and Leonard J. Savage. Moscati divides their debates into

\footnotetext{
${ }^{5}$ Recall from the previous paragraph that cardinal utility preserves only utility differences. Ratio-scale utility, instead, preserves also utility ratios.
} 
three time-spans. The first two are characterized by one underlying question: what does the von Neumann-Morgenstern expected utility function measure?

In the first period (1947-1950), there was a shared understanding of expected utility as a linear transformation of the risk-less (or Bernoulli) utility function ${ }^{6}$ introduced by the early marginalists. In the second phase (1950-1952), the interpretation of expected utility among the leading scholars at the time changed significantly. This was prompted by a rich exchange of letters initiated by Samuelson's request for feedback on an article (Samuelson 1950) in which he harshly criticized both the axioms underlying von Neumann and Morgenstern's expected utility theory and its empirical implications. The letter led to a heated epistolary discussion on what it is, exactly, that expected utility measures. In the end, all parties, apart from Allais, gradually came to accept the interpretation of expected utility advanced by Friedman in his correspondence with Baumol in the summer of 1950 (176). According to Friedman, expected utility is a monotonic transformation but not necessarily a linear transformation of risk-less (or Bernoulli) utility. Thus, while the two functions preserve ordinal information-they order alternatives in the same way-they need not preserve additional information, such as utility differences.

This common understanding of expected utility opened the third phase of the debate (1952-1955), which focused on the meaning of measuring utility per se. In that phase, numerous protagonists from the two previous phases (Friedman, Savage, Baumol) and new ones, such as Robert H. Strotz, Daniel Ellsberg, and Armen A. Alchian, wrote several articles proposing a novel notion of utility measurement. In these articles, they argued that measuring utility simply means assigning numbers to objects on the basis of a specific set of (non-mathematical or psychological) operations. They all stressed the conventional character of any method of measurement and clarified that the adoption of a specific measurement method depends, by and large, on its convenience for the purpose at hand. In the case of measuring utility, Moscati contends, the purpose, in those years, was perceived as describing and predicting behavior.

\footnotetext{
${ }^{6}$ The theorem by von Neumann and Morgenstern ([1944] 2007) states that if preferences over lotteries satisfy specific assumptions, then they can be represented by an expected utility function. An expected utility function represents the expected value of a lottery, where probabilities constitute a weight of the risk-less (or Bernoulli) utility function. A risk-less (or Bernoulli) utility function is defined on sure amounts. It carries the name of Daniel Bernoulli since he was the first to use a version of such a function. See, for example, Mas-Colell, Whinston, and Green $(1995,184)$.
} 
The fourth and final part of the book (1950-1985) differs slightly from the previous three. Indeed, it concentrates on the empirical attempts to measure utility rather than on its theoretical understanding. It offers a historical reconstruction of the laboratory experiments on measuring utility and the growing skepticism among economists about the possibility and significance of this endeavor. The reason for concentrating on the different empirical attempts to measure utility is that, after 1955, the discussion on the meaning of utility measurement was settled by widespread agreement on its interpretation. Indeed, as Moscati makes clear in the third part of the book (208-211), all economists who were working on the topic at that time (Friedman, Savage, Ellsberg, and others) finally converged on what measuring utility means. They all shared the view that measuring the utility of a specific option or object simply means assigning a number to that object according to a specific set of operations. Those numbers are, in fact, utilities and are assigned in a way that is arbitrary and conventional. This allowed economists to shift their attention to developing empirical techniques to estimate utility.

This last portion of the book is structured into two conceptually and historically distinct phases. The first (1950-1965) is mainly concerned with what Moscati labels the "age of confidence" (218). The phrase refers to the fact that, in those years, experiments performed to test expected utility theory were interpreted favorably, both by the experimenters and the wider economic academic community. This means that these experimental results were understood both as confirming the descriptive adequacy of expected utility theory and the possibility of empirically measuring expected utility. The second section considers the time when confidence in the possibility of measuring expected utility was replaced by skepticism (1965-1985). This skepticism was fueled by experiments showing that people tend to have choice patterns that resemble both the Allais and the Ellsberg Paradox. Those two 'paradoxes' were independently constructed by Maurice Allais and Daniel Ellsberg to describe choice situations in which people often make decisions that violate the predictions of expected utility theory. From the mid-1960s onwards, an increasing number of experiments have empirically demonstrated that people often do exhibit such 'paradoxical' behavior. These results, in turn, have undermined economists' confidence in the descriptive adequacy of expected utility theory.

Moscati ends part four, and the book itself, with the description of one specific experiment set up by economists John C. Hershey and Paul 
J. H. Schoemaker in 1985 (Hershey and Schoemaker 1985). In Moscati's view, this experiment, which provided empirical evidence that people display choice patterns that systematically violate expected utility theory, marks the birth of what later came to be known as 'behavioral economics'. With behavioral economics, a whole new historical chapter begins: the starting assumption is that experimental measurements of utility based on expected utility theory are flawed. This new turn in utility theory, Moscati concludes, generates many lines of research that would need a book all of its own.

\section{CRITICAl APPRAISAL}

Moscati has written an engaging narrative of the history of utility theory using a wide variety of debates, descriptions of exchanged letters, and biographical reconstructions. All these together have allowed Moscati to write an encompassing historical reconstruction of utility measurementthis constitutes one of the book's distinctive strengths. We thus find the text to be an indispensable companion to any researcher who is interested in utility theory.

In the remainder of this text, we want to reflect a bit more on an underlying idea that plays a key role in Moscati's historical analysis. Namely, the idea of a shared intellectual environment (as we label it)-both within the discipline of economics, which is Moscati's primary interest, and across disciplines, such as economics, psychology, and philosophy.

To start with, Moscati's reconstruction of the communication among economists deserves to be praised. He provides many descriptions of such communications, at least among some economists (see as an example the epistolary discussion at 176). For instance, as Moscati recognizes, there is a rich documented correspondence between Jevons and Walras, which would speak in favor of a shared conception of utility measurement, at least at a later stage of the development of their theories. Similarly, Moscati lays out in great detail how the acquaintance with the work of the first generation of marginalists influenced the work of the second generation.

We are, however, less persuaded by the idea of a shared intellectual environment across disciplines. To wit, a discussion of conceptions of measurement in fields outside economics is itself useful to the extent that economists were informed by the work carried out in those fields. Consider, for instance, the reference that Moscati makes to the "broad intellectual context" (24) within which discussions on measurement took place 
and his claim that it is "hardly surprising" that early marginalists conceived of measurement in the unit-based sense, given such an intellectual environment (24). While it is not necessary to demonstrate that the first and second generation of marginalists were well aware of the work that physicists, psychologists, and mathematicians were carrying out, Moscati's case would be buttressed by an explicit presentation of how specific ideas (in physics, psychology, etc.) came to be known in economics. However, this idea is not fully substantiated. For instance, the entire first chapter is dedicated to notions of measurement in different sciences, while in the second chapter there is a paragraph devoted to Jevons' work on measurement beyond utility (28); but, there is no explanation of how notions in other sciences influenced Jevons. The reader is left wondering whether Moscati assumes or has evidence for assuming that Jevons became acquainted with them thanks to his friendship with Edgeworth (53), who knew the mathematical work on measurement carried out by Voigt (23). However, Moscati does not provide a substantive clarification for his claim. Another example is the lack of documented evidence that the second generation of marginalists was exposed to the ideas of scholars working in other fields. These omissions leave the reader pondering whether the shared conception of measurement in a specific period was the result of a fortunate coincidence or of a deep-and conscious-shared intellectual understanding.

In conclusion, Measuring Utility is to be commended for its detailed reconstruction of the intellectual atmospheres during the history of utility measurement covered in the book. By recounting the years during which utility theory and the building blocks of modern economic analysis were developed, it improves greatly our understanding of what utility itself is and how it can be used, both from a descriptive and a normative point of view. This makes the book a recommended reading for all researchers who broadly deal with the concept of utility. Indeed, the turn that economics is taking nowadays, with the development of several different sub-branches, cannot be fully appreciated without a comprehensive picture of the different attempts at, and understandings of, utility measurement.

\section{REFERENCES}

Hershey, John C., and Paul J. H. Schoemaker. 1985. "Probability Versus Certainty Equivalence Methods in Utility Measurement: Are they Equivalent?” Management Science 31 (10): 1213-1231. 
Mas-Colell, Andreu, Michael D. Whinston, and Jerry R. Green. 1995. Microeconomic Theory. New York, NY: Oxford University Press.

Moscati, Ivan. 2007. "Early Experiments in Consumer Demand Theory: 1930-1970." History of Political Economy 39 (3): 359-401.

Moscati, Ivan. 2013a. "How Cardinal Utility Entered Economic Analysis: 1909-1944." The European Journal of the History of Economic Thought 20 (6): 906-939.

Moscati, Ivan. 2013b. "Were Jevons, Menger, and Walras Really Cardinalists? On the Notion of Measurement in Utility Theory, Psychology, Mathematics, and Other Disciplines, 1870-1910." History of Political Economy 45 (3): 373-414.

Moscati, Ivan. 2016a. "Measurement Theory and Utility Analysis in Suppes' Early Work, 1951-1958." Journal of Economic Methodology 23 (3): 252-267.

Moscati, Ivan. 2016b. "Measuring the Economizing Mind in the 1940s and 1950s: The Mosteller-Nogee and Davidson-Suppes-Siegel Experiments to Measure the Utility of Money." History of Political Economy 48 (suppl_1): 239-269.

Moscati, Ivan. 2016c. "Retrospectives: How Economists Came to Accept Expected Utility Theory: The Case of Samuelson and Savage.” Journal of Economic Perspectives 30 (2): 219-236.

Moscati, Ivan. 2017. "Expected Utility Theory and Experimental Utility Measurement, 1950-1985. From Confidence to Scepticism." The European Journal of the History of Economic Thought 24 (6): 1318-1354.

Samuelson, Paul A. 1950. "Probability and the Attempts to Measure Utility." Economic Review 1 (3): 167-173.

Von Neumann, John, and Oskar Morgenstern. (1944) 2007. Theory of Games and Economic Behavior. 60th Anniversary Commemorative Edition, with an introduction by Harold W. Kuhn and an afterword by Ariel Rubinstein. Princeton, NJ: Princeton University Press.

Annalisa Costella is a first-year PhD student at the Erasmus School of Philosophy. Her research interests include decision theory, axiomatic approaches to freedom of choice, and the welfare implications of behavioral economics.

Contact e-mail: <costella@esphil.eur.nl>

Enrico Mattia Salonia is enrolled in the first year of the doctoral track at the Toulouse School of Economics. His research interests are mainly in economic theory, in particular game and microeconomic theory.

Contact e-mail: <enrico-mattia.salonia@ut-capitole.fr> 